Pacific Journal of Mathematics

THE GENERALIZED SCHWARZ LEMMA FOR THE BERGMAN 


\title{
THE GENERALIZED SCHWARZ LEMMA FOR THE BERGMAN METRIC
}

\author{
MASAAKI SUZUKI
}

The function-theoretic criterion for the Bergman metric to be dominated by the Kobayashi metric on the domain in $\mathrm{C}^{n}$ is given. For this, we use the distinguished family of plurisubharmonic functions and $P$-metric of N. Sibony.

1. Introduction. Let $D$ be a hyperbolic domain in $\mathbf{C}^{n}$ (cf. Kobayashi [7]). On $D$ we can define some intrinsic metrics: the Carathéodory metric $C_{D}$, the Kobayashi metric $K_{D}$, and the Bergman metric $B_{D}$. It is known that $C_{D} \leq K_{D}$ and $C_{D} \leq B_{D}$. In this paper we investigate when the Bergman metric is dominated by the Kobayashi metric. Using N. Sibony's $P$-metric we give a function-theoretic criterion for the following condition (\#) to hold:

(\#) $B_{D} \leq c K_{D}$ on the tangent bundle of $D, c>0$ constant.

Under (\#) every holomorphic mapping $F: U \rightarrow D$ satisfies $F^{*} B_{D} \leq$ $2^{-1 / 2} c B_{U}$, where $U$ is the unit disc in $\mathbf{C}$ with the Bergman metric $B_{U}$. This theorem is called the generalized Schwarz lemma for the Bergman metric. According to N. Sibony [10], we introduce the family of functions

$$
\begin{array}{r}
S_{p}(D)=\left\{u: D \rightarrow[0,1) ; u(p)=0, C^{2}\right. \text {-class in a neighborhood } \\
\text { of } p \text { and } \log u \text { is plurisubharmonic in } D\} .
\end{array}
$$

Taking a Bergman kernel $k(z, \bar{w})$ of a domain $D$, we construct a function $\phi_{w}$ for a fixed point $w$ in $D$ as follows;

$$
\phi_{w}(z)=\phi_{w, \alpha}(z)=1-\left(\frac{|k(z, \bar{w})|^{2}}{k(z, \bar{z}) k(w, \bar{w})}\right)^{\alpha} \equiv 1-v^{\alpha}
$$

where $\alpha$ is a positive constant chosen for $D$. It is clear that $0 \leq \phi_{w} \leq 1$ and $\phi_{w}(w)=0$. Our main results are stated as follows.

(I) Let $D$ be a Bergman domain. If there is a constant $\alpha>0$ such that $\phi_{w}=\phi_{w, \alpha}$ belongs to $S_{w}(D)$ for each $w$ in $D$, then $B_{D} \leq \alpha^{-1 / 2} K_{D}$; hence $B_{D}$ satisfies the generalized Schwarz lemma. 
(II) If the Bergman metric $B_{D}$ of a domain $D$ satisfies the following condition; there exists a positive constant $\alpha$ such that, for each $w$ in $D$,

$(*) \quad \phi_{w}(z) B_{D}^{2}(z, \xi) \geq \alpha\left|\partial_{\xi} \log v(z)\right|^{2}$ for all $\xi \in \mathbf{C}^{n}$

$$
\text { and } z \in\{z \in D ; 0<v(z)<1\},
$$

then $B_{D} \leq \alpha^{-1 / 2} K_{D}$; therefore, $B_{D}$ satisfies the generalized Schwarz lemma.

In $\S 2$ we give some properties of the family $S_{p}(D)$. In $\S 3$ we arrange the basic properties of the intrinsic metrics, especially of the $P^{*}$-metric. In $\S 4$ we prove the main results.

In $\S 5$, for the classical domains, we construct the function $\phi_{w}$ and directly verify that each $\phi_{w}$ belongs to $S_{w}(D)$. Hence, we have the generalized Schwarz lemma for the classical domains (cf. Kobayashi [7, 8]).

The author is grateful to K. Azukawa for helpful discussions of this material.

2. The family $S_{p}(D)$. Though our argument is available on a complex manifold, we work mainly on a domain in $\mathbf{C}^{n}(n \geq 1)$.

Let $p$ be a fixed point of $D$ and

$$
\begin{aligned}
& S_{p}(D)=\left\{u: D \rightarrow[0,1) ; u(p)=0, C^{2}\right. \text {-class in a } \\
&\text { neighborhood of } p \text { and } \log u \text { is plurisubharmonic in } D\}, \\
& A_{p}(D)=\left\{u=|f|^{2} ; f \in \operatorname{Hol}(D, U), f(p)=0\right\},
\end{aligned}
$$

where $U$ is the unit disc in $\mathbf{C}$ and $\operatorname{Hol}(D, U)$ denotes the family of all holomorphic mappings $f: D \rightarrow U$. It is clear that $S_{p}(D) \supset A_{p}(D)$.

A nonnegative plurisubharmonic function $u$ such that $\log u$ is also plurisubharmonic $(\log 0=-\infty)$ is called a logarithmically plurisubharmonic function. Hereafter we abbreviate them p.s.h. and log.p.s.h. $S_{p}(D)$ is a special family of log.p.s.h. functions on $D$. We give some lemmas about the family $S_{p}(D)$.

LEMMA 2.1. (1) If $u_{1}, u_{2}$ belong to $S_{p}(D)$, then $r u_{1}+(1-r) u_{2} \in S_{p}(D)$ for any real number $r$ with $0 \leq r \leq 1$.

(2) If $f_{j}(j=1, \ldots, k)$ are holomorphic in $D$ and vanish at $p$ and $u(z)=\Sigma\left|f_{j}(z)\right|^{2}<1$ on $D$, then $u \in S_{p}(D)$.

Proof. (1) It is sufficient to prove that if $\log u_{j}(j=1,2)$ is subharmonic in an open set $G$ in $C$, then $\log \left(r_{1} u_{1}+r_{2} u_{2}\right)$ is subharmonic in $G$ for $r_{J} \geq 0$, with $r_{1}+r_{2}=1$. As in the book of Hörmander [4], we take a 
disc $G^{*} \subset G$ and a polynomial $P(t)(t \in \mathbf{C})$ such that $\log u \leq \operatorname{Re} P$ on $\partial G^{*}$, where $u=r_{1} u_{1}+r_{2} u_{2}$. Then $u \leq \exp (\operatorname{Re} P)$ on $\partial G^{*}$. Since $\log u_{J}-$ $\operatorname{Re} P$ is subharmonic in $G^{*}, r_{j} u_{j}|\exp (-P)|$ is also subharmonic in $G^{*}$. Hence $\left(r_{1} u_{1}+r_{2} u_{2}\right)|\exp (-P)|=u|\exp (-P)|$ is subharmonic in $G^{*}$. From $u|\exp (-P)| \leq 1$ on $\partial G^{*}$ and the maximum principle for the subharmonic function, we have $u|\exp (-P)| \leq 1$ on $G^{*}$, that is, $\log u \leq \operatorname{Re} P$ in $G^{*}$. Since $G^{*}$ is an arbitrary disc in $G, \log u$ is subharmonic in $G$.

(2) follows directly from (1).

The following lemma will be used in $\S 4$.

LEMMA 2.2. Let $v(z)$ be a $\mathscr{C}^{2}$-function on a domain $D$ with $0<v<1$.

(1) $1 / v(z)$ is $\log . p . s . h$. if and only if

$$
\left|\sum v_{i} \xi_{i}\right|^{2}-v \sum v_{i j} \xi_{i} \bar{\xi}_{j} \geq 0 \text { for all } \xi \in \mathbf{C}^{n}
$$

where $v_{i}=\partial v / \partial z_{l}, v_{j}=\partial v / \partial \bar{z}_{j}$ and $v_{\imath j}=\partial^{2} v / \partial z_{i} \partial \bar{z}_{j}$.

(2) The function $\phi(z)=1-v(z)^{\beta}$ ( $\beta$ is a positive constant) is log.p.s.h. if and only if

$$
\phi\left[\left|\sum v_{l} \xi_{l}\right|^{2}-v \sum v_{l j} \xi_{i} \bar{\xi}_{j}\right] \geq \beta\left|\sum v_{i} \xi_{i}\right|^{2} \quad \text { for all } \xi \in \mathbf{C}^{n} .
$$

We have

Proof. We show (2) only. It is clear that $(\log \phi)_{i j}=\left(\phi_{l j} \phi-\phi_{i} \phi_{j}\right) \phi^{-2}$.

$$
\phi_{i}=-\beta v^{\beta-1} v_{i} \text { and } \phi_{i j}=\beta v^{\beta-2}\left((1-\beta) v_{i} v_{j}-v v_{i j}\right) .
$$

Therefore,

$$
\phi_{i j} \phi-\phi_{i} \phi_{j}=\beta v^{\beta-2}\left[(\phi-\beta) v_{i} v_{j}-\phi v v_{i j}\right] .
$$

Hence (2.3) is equivalent to the fact that the matrix $\left[(\log \phi)_{i j}\right]$ is positive semidefinite.

3. The intrinsic metrics. Let $M$ be a complex manifold and $T M$ its holomorphic tangent bundle. According to Kobayashi [8], we call a function $X=X_{M}(p, \xi)$ on $T M$ a complex Finsler metric on $M$ when it satisfies the following conditions;

(i) $X_{M}(p, \xi)$ is an upper semicontinuous positive function on $T M$,

(ii) $X_{M}(p, \lambda \xi)=|\lambda| X_{M}(p, \xi)$ for any $\lambda \in \mathbf{C}$.

The intrinsic metric is the biholomorphic invaraiant complex Finsler metric which is determined only by the complex analytic structure of the complex manifold. As examples of intrinsic metrics, there are the Carathéodory, the Kobayashi, and the Bergman metrics. In this section we 
give the basic properties of them and introduce a new intrinsic metric, that is, the $P^{*}$-metric (cf. [10] and [14]).

When $M$ is a domain in $\mathbf{C}^{n}$, since $T D \cong D \times \mathbf{C}^{n}$, we may assume a tangent vector $\xi \in \mathbf{C}^{n}$. Let $\left(z_{1}, \ldots, z_{n}\right)$ be the canonical coordinates of $\mathbf{C}^{n}$, and let $(p, \xi)$ denote a pair of $T D$.

The Carathéodory metric (C-metric) $C_{D}$ of a domain $D$ is given by

$$
C_{D}(p, \xi)=\sup \left\{\left|\partial_{\xi} f(p)\right| ; f \in \operatorname{Hol}(D, U), f(p)=0\right\}
$$

where $\partial_{\xi} f(p)=\sum_{i} \partial f / \partial z_{i}(p) \xi_{i}$.

The Kobayashi metric (K-metric) $K_{D}$ of $D$ is defined by

$$
K_{D}(p, \xi)=\inf \left\{1 / r ; F \in \operatorname{Hol}(U, D), F(0)=p, F^{\prime}(0)=r \xi, r>0\right\} \text {. }
$$

Though they are not always positive, we mainly work on hyperbolic domains. The following theorem is well known.

TheOREM $3.1[7,8]$. Let $D$ be a hyperbolic domain in $\mathrm{C}^{n}$.

(1) The $C$-metric is continuous on TD and the K-metric is upper semicontinuous on $T D$.

(2) The $C$ and $K$-metrics are decreasing for any holomorphic mappings: for $F \in \operatorname{Hol}(D, E)(D, E$ are domains $)$,

$$
\begin{aligned}
& C_{E}\left(F(p), F^{\prime}(p) \xi\right) \leq C_{D}(p, \xi), \\
& K_{E}\left(F(p), F^{\prime}(p) \xi\right) \leq K_{D}(p, \xi) .
\end{aligned}
$$

N. Sibony [10] introduced the $P$-metric as follows:

$$
P_{D}(p, \xi)=\sup \left\{L(u ; p, \xi)^{1 / 2} ; u \in S_{p}(D)\right\},
$$

where

$$
L(u ; p, \xi)=\sum \frac{\partial^{2} u}{\partial z_{i} \partial \bar{z}_{j}}(p) \xi_{i} \bar{\xi}_{j}
$$

is the Levi form of $u . P_{D}$ is locally integrable, but its upper semicontinuity is unknown yet. We define

$$
P_{D}^{*}(p, \xi)=\lim \sup P_{D}(q, \zeta) \text { as }(q, \zeta) \rightarrow(p, \xi) .
$$

Then $P_{D}^{*}$ is upper semicontinuous and $P_{D} \leq P_{D}^{*}$ and $P_{U}^{*}(0,1)=1$ for the unit disc $U$ in C. $P_{D}^{*}$ is called the $P^{*}$-metric of $D$.

LeMma 3.2 (cf. [10], [14]). The $P^{*}$-metric is an intrinsic metric having the decreasing property for holomorphic mappings, and $C_{D} \leq P_{D}^{*} \leq K_{D}$ on $T D$. 
Proof. The first half follows from the result of Sibony [10] (see also [14]). We give a simple proof for the last half. Noting that $S_{p}(D) \supset A_{p}(D)$ and $L\left(|f|^{2} ; p, \xi\right)=\left|\partial_{\xi} f(p)\right|^{2}$, we have $C_{D} \leq P_{D}^{*}$. Taking a mapping $F$ in $\operatorname{Hol}(U, D)$ with $F(0)=p, F^{\prime}(0)=r \xi(r>0)$, from the decreasing property for $F$, we have

$$
P_{D}^{*}(p, r \xi)=P_{D}^{*}\left(F(0), F^{\prime}(0)\right) \leq P_{U}^{*}(0,1)=1 .
$$

Therefore, $P_{D}^{*}(p, \xi) \leq 1 / r$. Since the $\mathrm{K}$-metric is the infimum of such $1 / r$, we obtain the inequality $P_{D}^{*} \leq K_{D}$.

Let $X_{D}(p, \xi)$ be one of the intrinsic metrics on a domain $D$. A point $p$ in $D$ is said to be an $X$-hyperbolic point if there is a neighborhood $V$ of $p$ and a constant $c>0$ such that $X_{D}(q, \xi) \geq c\|\xi\|$ for all $\xi \in \mathbf{C}^{n}$ at every point $q$ in $V$, where \|\| is the Euclidean norm of $\mathbf{C}^{n}$. If each oint of $D$ is $X$-hyperbolic point, then $D$ is said to be an $X$-hyperbolic domain.

4. The generalized Schwarz lemma. Let $D$ be a domain in $\mathbf{C}^{n}$. The reproducing kernel $k(z, \bar{w})$ of the Hilbert space $L^{2} H(D)$ of $L^{2}$-holomorphic functions on $D$ is called the Bergman kernel of $D$. It is holomorphic in $D \times \bar{D}$ (where $\bar{D}$ is the complex conjugate of $D$.) Defining

$$
B_{D}^{2}(z, \xi)=\sum\left(\frac{\partial^{2}}{\partial z_{i} \partial \bar{z}_{j}} \log k(z, \bar{z})\right) \xi_{i} \bar{\xi}_{j}, \quad \xi \in \mathbf{C}^{n},
$$

we have the Bergman metric $B_{D}$ of $D$ provided that the right side of the above is positive for all $\xi \neq 0$. The Bergman metric is a Kähler metric and an intrinsic metric, but does not always have the decreasing property for holomorphic mappings.

We call a domain $D$ with $B_{D} \neq 0$ the Bergman domain.

For the upper semicontinuous Finsler metric, the holomorphic curvature is defined (cf. [12]) and coincides with the holomorphic sectional curvature if the metric is $C^{2}$-hermitian. Let $U$ be the unit disc in $\mathbf{C}$ with the canonical metric $\left(1-|t|^{2}\right)^{-2}|d t|^{2}$. The following lemma is a generalization of the Schwarz lemma (cf. [7, III, Theorem 2.1]) to the upper semicontinuous case.

LEMMA $4.1\left(c f .[14]\right.$.) Let $X_{M}(p, \xi)$ be a complex Finsler metric on a $K$-hyperbolic manifold $M$. If its holomorphic curvature is bounded from above by a negative constant $-c^{2}(c>0)$, then $X_{M} \leq 2 c^{-1} K_{M}$, and for any holomorphic mapping $F: U \rightarrow M, X_{M}\left(F(t), F^{\prime}(t)\right) \leq 2 c^{-1} K_{U}(t, 1)$.

Since the holomorphic curvature coincides with the holomorphic sectional curvature for the Bergman metric, we have the following lemma. 
Lemma 4.2. Let $D$ be a Bergman domain. If the holomorphic sectional curvature of the Bergman metric $B_{D}$ of $D$ is bounded from above by $a$ negative constant $-k$, then $B_{D} \leq 2 k^{-1 / 2} K_{D}$, and for every holomorphic mapping $F: U \rightarrow D$,

$$
B_{D}\left(F(t), F^{\prime}(t)\right) \leq(2 / k)^{1 / 2} B_{U}(t, 1), \quad t \in U .
$$

Proof. The first half follows from Lemma 4.1. For any holomorphic mapping $F: U \rightarrow D$, we have

$$
\begin{aligned}
B_{D}\left(F(t), F^{\prime}(t)\right) & \leq 2 k^{-1 / 2} K_{D}\left(F(t), F^{\prime}(t)\right) \\
& \leq 2 k^{-1 / 2} K_{U}(t, 1)=(2 / k)^{1 / 2} B_{U}(t, 1),
\end{aligned}
$$

since $\sqrt{2} K_{U}=B_{U}$.

From the proof of this lemma, we see that if the Bergman metric is dominated by the Kobayashi metric, then any $F \in \operatorname{Hol}(U, D)$ is decreasing with respect to the Bergman metric (i.e. (*) holds).

For a domain $D$ in $\mathbf{C}^{n}$, we consider the following condition:

(\#) $B_{D} \leq c K_{D}$ on $T D$ for some constant $c>0$.

(A) If $D$ is a bounded homogeneous domain, then $C_{D}, K_{D}, P_{D}^{*}$ and $B_{D}$ are all equivalent metrics because of their biholomorphic invariantness and the homogeneity of $D$. Especially, (\#) is satisfied.

(B) If the Bergman metric of $D$ has strictly negative holomorphic sectional curvature, then by Lemma 4.2, (\#) is satisfied.

Here we shall give a function-theoretic criterion for (\#). Let $D$ denote the Bergman domain in $\mathbf{C}^{n}$ with the Bergman kernel $k(z, \bar{w})$. For a fixed point $w$ in $D$, we construct a function on $D$ by

$$
\phi_{w}(z)=\phi_{w, \alpha}(z)=1-\left(\frac{|k(z, \bar{w})|^{2}}{k(z, \bar{z}) k(w, \bar{w})}\right)^{\alpha},
$$

where $\alpha$ is a positive constant properly chosen for $D$. When $\alpha=1 / 2$, this is a square of the invariant distance $\rho_{D}(z, w)$ of Skwarczynski (cf. [11]).

LEMMA 4.3. With the above notations, the following hold.

(1) $0 \leq \phi_{w} \leq 1$ and $\phi_{w}(w)=0$.

(2) If $k(z, \bar{w}) \neq 0$, then $L\left(\phi_{w} ; w, \xi\right)=\alpha B_{D}^{2}(w, \xi)$.

(3) $\phi_{w}$ is biholomorphically invariant, i.e. for any biholomorphic automorphism $g$ of $D, \phi_{g(w)}(g(z))=\phi_{w}(z)$.

Proof. (1) is clear. (3) follows from the fact that

$$
k(g(z), \overline{g(w)}) J_{g}(z) \overline{J_{g}(w)}=k(z, \bar{w}),
$$


where $J_{g}$ is the Jacobian determinant of $g$. It remains to show (2). By simple calculations we have

$$
\begin{aligned}
\partial^{2} \phi_{w} / \partial z_{i} \partial \bar{z}_{j}(w) & =\alpha k(w, \bar{w})^{-2}\left\{k_{i j}(w, \bar{w}) k(w, \bar{w})-k_{l}(w, \bar{w}) k_{j}(w, \bar{w})\right\} \\
& =\alpha\left(\partial^{2} \log k / \partial z_{i} \partial \bar{z}_{j}\right)(w) .
\end{aligned}
$$

Hence, $L\left(\phi_{w} ; w, \xi\right)=\alpha B_{D}^{2}(w, \xi)$.

A domain $D$ in $\mathbf{C}^{n}$ is called a Lu Qi-keng domain if $k(z, \bar{w}) \neq 0$ for all $z, w$ in $D$.

Theorem 4.4. Let $D$ be a Bergman domain in $\mathbf{C}^{n}$. If there is positive constant $\alpha$ such that $\phi_{w}=\phi_{w, \alpha}$ belongs to $S_{w}(D)$ for each $w$ in $D$, then $D$ is a Lu Qi-keng domain and

$$
B_{D}(w, \xi) \leq \alpha^{-1 / 2} P_{D}^{*}(w, \xi) \leq \alpha^{-1 / 2} K_{D}(w, \xi) \quad \text { on } T D .
$$

Proof. When $\phi_{w}$ belongs to $S_{w}(D)$ for each $w$ in $D, \phi_{w}$ is a p.s.h. function. Hence, by the maximum principle, $0 \leq \phi_{w}<1$ in $D$, that is, $k(z, w) \neq 0$ for all $z, w$ in $D$. So $D$ is a Lu Qi-keng domain. From the definition of $P^{*}$-metric and Lemma 4.3(2), we have

$$
\alpha^{1 / 2} B_{D} \leq P_{D}^{*} \leq K_{D} \text { on } T D \text {. }
$$

Using Lemma 2.2 we can rewrite the condition that $\phi_{w}$ belongs to $S_{w}(D)$ for each $w$ in $D$. For a fixed point $w$ in $D$, let $v(z)=$ $|k(z, \bar{w})|^{2} / k(z, \bar{z}) k(w, \bar{w})$. We remark that $k(z, \bar{w})$ is holomorphic in $z \in D$. We set

$$
\begin{aligned}
& A_{w}=\{z \in D ; v(z)=0\}=\{z \in D ; k(z, \bar{w})=0\}, \\
& E_{w}=\{z \in D ; v(z)=1\}=\left\{z \in D ; \phi_{w}(z)=0\right\}, \\
& D_{w}=D \backslash\left(A_{w} \cup E_{w}\right) .
\end{aligned}
$$

Then $A_{w}$ is an analytic set in $D$, and $E_{w}$ contains at least $w$.

Proposition 4.5. For fixed $w$ in $D, \phi_{w}=1-v^{\alpha}$ is log.p.s.h. if and only if the following inequality holds:

$$
\phi_{w}(z) B_{D}^{2}(z, \xi) \geq \alpha\left|\partial_{\xi} \log v(z)\right|^{2}
$$

for all $\xi \in \mathbf{C}^{n}-\{0\}$ at all $z \in D_{w}$.

Proof. Since $0<v<1$ on $D_{w}$, we may verify that (2.3) in Lemma 2.2 is equivalent to (4.6) (we set $\beta=\alpha$ ). Assume that this was proved. Then 
$\log \phi_{w}$ is p.s.h. in $D_{w}$. For $z$ in $E_{w}$, we set $\log \phi_{w}(z)=-\infty$. Hence $\log \phi_{w}$ is p.s.h. in $D \backslash A_{w}$. Remarking that $\log \phi_{w}$ is negative in $D$ and $A_{w}$ is the analytic set in $D$, we can extend $\log \phi_{w}$ plurisubharmonically to $D$ (by the p.s.h. extension theorem of Grauert and Remmert). Thus it remains to show that (2.3) is equivalent to (4.6) for each $w$ in $D$. For a fixed $w$ in $D$, we write $\phi=\phi_{w}, k=k(z, \bar{z}), h=k(z, \bar{w}), a=k(w, \bar{w})$. Then $v=h \bar{h} / a k$, $\phi=1-(h \bar{h} / a k)^{\alpha}$. By partial differentiation we have the following:

$$
\begin{aligned}
v_{i} & =\bar{h}\left(h_{i} k-h k_{i}\right) / a k^{2}, \\
v_{i j} & =\left\{h_{i} \bar{h}_{j} k^{2}-\left(\bar{h} h_{i} k_{j}+h \bar{h}_{j}+h \bar{h} k_{i j}\right) k+2 h \bar{h} k_{i} k_{j}\right\} / a k^{3} .
\end{aligned}
$$

Substituting these in

$$
A \equiv \phi\left|\sum v_{i} \xi_{i}\right|^{2}-\phi v \sum v_{i j} \xi_{i} \bar{\xi}_{j}-\alpha\left|\sum v_{i} \xi_{i}\right|^{2}
$$

we get

$$
\begin{aligned}
A & =\phi v^{2}\left\{k \sum k_{i j} \xi_{i} \bar{\xi}_{j}-\left|\sum k_{i} \xi_{i}\right|^{2}\right\} / k^{2}-\alpha v^{2}\left|\sum \frac{v_{i}}{v} \xi_{i}\right|^{2} \\
& =v^{2}\left\{\phi B_{D}^{2}(z, \xi)-\alpha\left|\partial_{\xi} \log v\right|^{2}\right\} .
\end{aligned}
$$

Therefore (2.3) holds if and only if (4.6) holds because of $0<v<1$ on $D_{w}$.

THEOREM 4.7. If the Bergman metric $B_{D}$ of a Bergman domain $D$ satisfies the following condition: for each $w$ in $D$ there is a positive constant $\alpha$ such that (4.6) holds, then $B_{D} \leq \alpha^{-1 / 2} K_{D}$. Hence every holomorphic mapping $F$ : $U \rightarrow D$ satisfies $F^{*} B_{D} \leq(2 \alpha)^{-1 / 2} B_{U}$.

Proof. By Proposition 4.5 we have $\phi_{w} \in S_{w}(D)$ for each $w$ in $d$. From Theorem 4.4 the conclusion is obtained.

Also, for any $F \in \operatorname{Hol}(U, D)$.

$$
\begin{aligned}
B_{D}\left(F(t), F^{\prime}(t)\right) & \leq \alpha^{-1 / 2} K_{D}\left(F(t), F^{\prime}(t)\right) \\
& \leq \alpha^{-1 / 2} K_{U}(t, 1)=(2 \alpha)^{-1 / 2} B_{U}(t, 1),
\end{aligned}
$$

since $B_{U}=2^{1 / 2} K_{U}$.

REMARKs. (1) $E_{w}$ is the polar set of $\log \phi_{w}$. If the coordinate functions $z_{1}, \ldots, z_{n}$ are in $L^{2} H(D)$ and the volume of $D$ is finite, then $E_{w}=\{w\}$.

(2) There exist the bounded homogeneous domains in $\mathbf{C}^{n}(n \geq 7)$ of which the Bergman metrics have positive holomorphic sectional curvatures (cf. D’Atri [2]). 
(3) There is a bounded pseudoconvex domain in $\mathbf{C}^{3}$ with $C^{\infty}$-boundary, which does not satisfy condition (\#) (cf. Diederich-Fornaess [3]).

(4) The annulus $A=\{t \in \mathbf{C} ; r<|t|<1\}$ is not the Lu Qi-keng domain. Therefore $\log \phi_{w}$ is not p.s.h. for some $w \in A$ (cf. Skwarczynski [11]).

5. The classical domains. In this section we construct the function $\phi_{w}$ for the classical domains (the bounded symmetric domains of four main type) $R$ and directly verify that they belong to $S_{w}(R)$ for some properly chosen $\alpha$.

We begin with the unit ball.

EXAmple 5.1. Let $D$ be the unit ball $B_{n}=\left\{z \in \mathbf{C}^{n} ;\|z\|<1\right\}$. Then its Bergman kernel is $k(z, \bar{w})=c_{n}(1-z \cdot \bar{w})^{-n-1}$, where $c_{n}=n ! \pi^{-n}$. Let 0 be the origin of $\mathbf{C}^{n}$. Taking $\alpha=1 /(n+1)$ we have $\phi_{0}(z)=\|z\|^{2}$ for $w=0$. It is clear that $\phi_{0} \in S_{0}\left(B_{n}\right)$. For any other point $w$ in $B_{n}$, taking an automorphism $g$ of $B_{n}$ with $g(0)=w$, we have $\phi_{w}(z)=\phi_{0}(g(z))=$ $\|g(z)\|^{2}$, which belongs to $S_{w}\left(B_{n}\right)$, and

$$
1-\left(1-\|z\|^{2}\right)\left(1-\|w\|^{2}\right) /\left|1-z \cdot \bar{w}^{2}\right|=\|g(z)\|^{2} .
$$

The last formula is well known (cf. Rudin [9], p. 26).

The four classical domains $R_{\mathrm{I}}, R_{\mathrm{II}}, R_{\mathrm{III}}$, and $R_{\mathrm{IV}}$, are given as follows ( $M(m, n)$ denotes the set of all $m \times n$ matrices):

$$
\begin{aligned}
R_{\mathrm{I}} & =\left\{Z \in M(m, n) ; I_{n}-Z^{*} Z>0\right\}, \\
R_{\mathrm{II}} & =\left\{Z \in M(n, n) ; Z^{\prime}=Z, I_{n}-Z^{*} Z>0\right\}, \\
R_{\mathrm{III}} & =\left\{Z \in M(n, n) ; Z^{\prime}=-Z, I_{n}-Z^{*} Z>0\right\}, \\
R_{\mathrm{IV}} & =\left\{z \in \mathbf{C}^{n} ;\left|\sum z_{i}^{2}\right|^{2}+1-2\|z\|^{2}>0,\left|\sum z_{i}^{2}\right|<1\right\},
\end{aligned}
$$

where $I_{n}$ is the $n \times n$ unit matrix and $Z^{\prime}$ is the transpose of $Z$ and $Z^{*}=\bar{Z}^{\prime}$.

Let $R$ or $R_{j}$ denote one of these domains, and 0 be the zero matrix or the origin of $\mathbf{C}^{n}$.

Proposition 5.2. (1) For $R_{\mathrm{I}}$, choosing $\alpha=1 /(m+n) n$, we set

$$
\phi_{0}(Z)=1-\left(\operatorname{det}\left(I_{n}-Z^{*} Z\right)\right)^{1 / n},
$$

(2) For $R_{\mathrm{II}}$, choosing $\alpha=1 /(n+1) n$, we set

$$
\phi_{0}(Z)=1-\left(\operatorname{det}\left(I_{n}-Z^{*} Z\right)\right)^{1 / n},
$$


(3) For $R_{\text {III }}$, choosing $\alpha=1 /(n-1)[n / 2]$, we set

$$
\phi_{0}(Z)=1-\left(\operatorname{det}\left(I_{n}-Z^{*} Z\right)\right)^{[n / 2]} \text {, }
$$

(4) For $R_{\text {IV }}$, choosing $\alpha=1 / 2 n$, we set

$$
\phi_{0}(z)=1-\left(1+\left|\sum z_{i}^{2}\right|^{2}-2\|z\|^{2}\right)^{1 / 2} .
$$

Then each $\phi_{0}$ is log.p.s.h., hence it belongs to $S_{0}(R)$; also $\phi_{w}(Z)=\phi_{0}(g(Z))$ belongs to $S_{w}(R)$ for any point $w$ in $R$, where $g$ is an automorphism of $R$ with $g(w)=0$.

We quote two lemmas to prove this proposition.

LeMma 5.3 ([7], p. 34). Let $B_{J}$ be the Bergman metric of $R_{j}(j=\mathrm{I}$, II, III, IV). Then

$$
\begin{aligned}
& B_{\mathrm{I}}^{2}(Z, \xi)=(m+n) \operatorname{Trace}\left[\left(I_{n}-Z^{*} Z\right)^{-1} \xi\left(I_{m}-Z Z^{*}\right)^{-1} \xi^{*}\right], \\
& B_{\mathrm{II}}^{2}(Z, \xi)=(n+1) \operatorname{Trace}\left[\left(I_{n}-Z^{*} Z\right)^{-1} \xi\left(I_{n}-Z Z^{*}\right)^{-1} \xi^{*}\right], \\
& B_{\mathrm{III}}^{2}(Z, \xi)=(n-1) \operatorname{Trace}\left[\left(I_{n}-Z^{*} Z\right)^{-1} \xi\left(I_{n}-Z Z^{*}\right)^{-1} \xi^{*}\right], \\
& B_{\mathrm{IV}}^{2}(z, \xi)=2 n A^{-2} \xi\left[A\left(I_{n}-2 z^{\prime} \bar{z}\right)+2\left(I_{n}-z^{\prime} \bar{z}\right) z^{*} z\left(I_{n}-z^{\prime} \bar{z}\right)\right] \xi^{*},
\end{aligned}
$$

where

$$
A=\left|\sum z_{i}^{2}\right|^{2}+1-2\|z\|^{2}
$$

Lemma 5.4 ([5]). (1) For $Z \in R_{\mathrm{I}}$, there exists an element $g$ of $G_{0}$ (the isotropy subgroup of automorphism group of $R_{\mathrm{I}}$ ) such that

$$
g Z=\left(\begin{array}{cccc}
\lambda_{1} & & \\
& & & 0 \\
& & \ddots & \\
& & & \lambda_{n}
\end{array}\right), \quad 1>\lambda_{1} \geq \cdots \geq \lambda_{n} \geq 0, m \geq n .
$$

(2) For $Z \in R_{\mathrm{II}}$, there is a $g \in G_{0}$ such that

$$
g Z=\operatorname{diagonal}\left[\lambda_{1}, \ldots, \lambda_{n}\right], \quad 1>\lambda_{1} \geq \cdots \geq \lambda_{n} \geq 0 .
$$

(3) For $Z \in R_{\mathrm{III}}$, there is a $g \in G_{0}$ such that

$$
g Z=\text { diagonal }\left[\left(\begin{array}{cc}
0 & \lambda_{1} \\
-\lambda_{1} & 0
\end{array}\right), \ldots,\left(\begin{array}{cc}
0 & \lambda_{k} \\
-\lambda_{k} & 0
\end{array}\right),(0)\right],
$$

where $k=[n / 2]$, and the last term is 0 if $n$ is odd. 
(4) For $z \in R_{\mathrm{IV}}$, there is a $g \in G_{0}$ such that

$$
g z=(\lambda, i \mu, 0, \ldots, 0), \quad \lambda, \mu \text { real numbers. }
$$

Proof of Proposition 5.2. Since

$$
\phi_{0}(Z)=1-\left(|k(Z, 0)|^{2} /(k(Z, \bar{Z}) k(0,0))\right)^{\alpha}
$$

and $k(Z, 0)$ is a constant, each $\phi_{0}$ is as above. (For the Bergman kernel of the classical domains, see, for example, [7, p. 34].) We directly verify (4.6) for the domain $R_{\mathrm{I}}(m \geq n)$. Note that $R_{\mathrm{I}}$ is a Lu Qi-keng domain; thus $A_{0}$ is the empty set and $E_{0}$ is point 0 only. From the biholomorphic invariantness of $\phi_{0}$ and Lemma 5.4, it is sufficient to show (4.6) at the point

$$
\begin{aligned}
& Z_{0}=\left(\begin{array}{cccc}
\lambda_{1} & & & \\
& & \ddots & \\
& & \ddots & \\
& 0 & & \\
& & & \lambda_{n}
\end{array}\right) \quad\left(1>\lambda_{1} \geq 0\right) . \\
& V\left(Z_{0}\right)^{\alpha}=\prod_{1}^{n}\left(1-\lambda_{J}^{2}\right)^{1 / n} ;
\end{aligned}
$$

thus

$$
\phi_{0}\left(Z_{0}\right)=1-\prod_{1}^{n}\left(1-\lambda_{j}^{2}\right)^{1 / n}
$$

From Lemma 5.3 we have

$$
B_{D}^{2}\left(Z_{0}, \xi\right)=(m+n)\left[\sum_{i, j}^{n} \frac{\left|\xi_{i j}\right|^{2}}{\left(1-\lambda_{i}^{2}\right)\left(1-\lambda_{j}^{2}\right)}+\sum_{i=1}^{n} \sum_{k>n}^{m} \frac{\left|\xi_{k i}\right|^{2}}{1-\lambda_{l}^{2}}\right]
$$

By partial differentiation

$$
\frac{\partial}{\partial z_{\mu \nu}} \operatorname{det}\left(I_{n}-Z^{*} Z\right)=(-1)^{\mu+1} \delta_{\mu \nu} \lambda_{\mu} \prod_{i \neq \mu}\left(1-\lambda_{i}^{2}\right) \quad \text { at } Z=Z_{0} .
$$

Hence,

$$
\begin{aligned}
\alpha\left|\partial_{\xi} \log v\left(Z_{0}\right)\right|^{2}=\frac{m+n}{n}\left|\partial_{\xi} \log \operatorname{det}\left(I_{n}-Z_{0}^{*} Z_{0}\right)\right|^{2} \\
=\frac{m+n}{n}\left|\sum_{\mu, \nu} \frac{\partial}{\partial z_{\mu \nu}}\left(\operatorname{det}\left(I_{n}-Z_{0}^{*} Z_{0}\right) \xi_{\mu \nu}\right)\right|^{2}\left|\operatorname{det}\left(I_{n}-Z_{0}^{*} Z_{0}\right)\right|^{-2} \\
=\frac{m+n}{n}\left|\sum_{\mu} \frac{\lambda_{\mu}}{1-\lambda_{\mu}^{2}} \xi_{\mu \mu}\right|^{2} .
\end{aligned}
$$


Let $T\left(Z_{0}\right)$ be the difference of the left and right sides of (4.6) at $Z_{0}$. Then

$$
\begin{aligned}
T\left(Z_{0}\right)= & (m+n)\left(1-\prod_{j}\left(1-\lambda_{j}^{2}\right)^{1 / n}\right) \\
& \cdot\left(\sum_{i, j} \frac{\left|\xi_{i j}\right|^{2}}{\left(1-\lambda_{i}^{2}\right)\left(1-\lambda_{j}^{2}\right)}+\sum_{i} \sum_{k>n} \frac{\left|\xi_{k i}\right|^{2}}{1-\lambda_{i}^{2}}\right) \\
& -\frac{m+n}{n}\left|\sum_{\mu} \frac{\lambda_{\mu}}{1-\lambda_{\mu}^{2}} \xi_{\mu \mu}\right|^{2} .
\end{aligned}
$$

Noting that $\Pi\left(1-\lambda_{j}^{2}\right)^{1 / n} \leq 1-(1 / n) \sum \lambda_{j}^{2}$, we have

$$
\begin{aligned}
T\left(Z_{0}\right) \geq & \frac{m+n}{n}\left(\sum \lambda_{j}^{2}\right)\left(\sum\left|\xi_{i j}\right|^{2}\left(1-\lambda_{i}^{2}\right)^{-1}\left(1-\lambda_{j}^{2}\right)^{-1}\right) \\
& -\frac{m+n}{n}\left|\sum \lambda_{\mu} \xi_{\mu \mu}\left(1-\lambda_{\mu}^{2}\right)^{-1}\right|^{2} \geq 0
\end{aligned}
$$

by Schwarz' inequality.

It is the same for (2) and (3) as (1). For $R_{\text {IV }}$ we may verify (2.3) directly at $(\lambda, i \mu, 0, \ldots, 0)$. The proof is reduced to the following inequality;

$$
\begin{aligned}
(\phi-1 / 2)[ & \lambda^{2}\left(\lambda^{2}-\mu^{2}-1\right)^{2}\left|\xi_{1}\right|^{2}+\mu^{2}\left(\lambda^{2}-\mu^{2}+1\right)\left|\xi_{2}\right|^{2} \\
& \left.+i \lambda \mu\left(\lambda^{2}-\mu^{2}-1\right)\left(\lambda^{2}-\mu^{2}+1\right)\left(\bar{\xi}_{1} \xi_{2}-\xi_{1} \bar{\xi}_{2}\right)\right] \\
& -2 \phi v\left[\left(2 \lambda^{2}-1\right)\left|\xi_{1}\right|^{2}+\left(2 \mu^{2}-1\right)\left|\xi_{2}\right|^{2}+2 i \lambda \mu\left(\bar{\xi}_{1} \xi_{2}-\xi_{1} \bar{\xi}_{2}\right)\right] \\
& +2 \phi v\left(\sum_{3}^{n}\left|\xi_{l}\right|^{2}\right) \\
\geq & 0 \quad \text { for all } \xi \in \mathbf{C}^{n},
\end{aligned}
$$

where $v=\left|\lambda^{2}-\mu^{2}\right|^{2}+1-2\left(\lambda^{2}+\mu^{2}\right)(0<v<1), \phi=1-v^{1 / 2}$, and $\left|\lambda^{2}-\mu^{2}\right|<1$. Simple but long calculations show that the above inequality holds.

$$
\begin{array}{r}
\text { COROLlaRY 5.5. } B_{\mathrm{I}}^{2} \leq(m+n) n K_{\mathrm{I}}^{2}, B_{\mathrm{II}}^{2} \leq(n+1) n K_{\mathrm{II}} \\
B_{\mathrm{III}}^{2} \leq(n-1)[n / 2] K_{\mathrm{III}}^{2}, \quad B_{\mathrm{IV}}^{2} \leq 2 n K_{\mathrm{IV}}^{2}
\end{array}
$$


Proof. From Theorem 4.4 and Proposition 5.2, we have, for example,

$$
B_{\mathrm{I}}^{2}(0, \xi) \leq(m+n) n K_{\mathrm{I}}^{2}(0, \xi) \text { for all } \xi \in \mathbf{C}^{n} .
$$

Using the homogeneity of $R_{j}$ and invariantness of the Bergman and Kobayashi metrics, we have the conclusion.

Remarks. For the classical domains $R_{j}, C, P^{*}$, and $\mathrm{K}$-metrics all coincide. The $\mathrm{K}$-metric $K_{\mathrm{I}}(0, \xi)$ is given by

$$
K_{\mathrm{I}}^{2}(0, \xi)=\max \left\{\text { eigenvalues of } \xi^{*} \xi\right\}
$$

(cf. [13]). Hence,

$$
K_{\mathrm{I}}^{2}(0, \xi) \geq n^{-1} \text { Trace } \xi^{*} \xi=(m+n)^{-1} n^{-1} B_{\mathrm{I}}^{2}(0, \xi)=\alpha B_{\mathrm{I}}^{2}(0, \xi) .
$$

On the other hand, the holomorphic sectional curvature $\kappa$ of $B_{\mathrm{I}}^{2}$ satisfies $-4 /(m+n) \leq \kappa \leq-4 /(m+n) n$, hence the constant $k$ in Lemma 4.2 is equal to $4 \alpha$ and we have $\alpha B_{\mathrm{I}}^{2} \leq K_{\mathrm{I}}^{2}$. For $R_{\mathrm{II}}$ and $R_{\mathrm{III}}$, we can do similarly. With respect to $R_{\mathrm{IV}}$,

$$
K_{\mathrm{IV}}^{2}(0, \xi)=\|\xi\|^{2}+\left(\|\xi\|^{4}-\left|\xi^{\prime} \xi\right|^{2}\right)^{1 / 2} \geq\|\xi\|^{2}=1 / 2 n B_{\mathrm{IV}}^{2}(0, \xi),
$$

and the holomorphic sectional curvature $\kappa$ of $B_{\mathrm{IV}}^{2}$ satisfies $-4 / n \leq \kappa \leq$ $-2 / n$. Hence $\alpha B_{\mathrm{IV}}^{2} \leq K_{\mathrm{IV}}^{2}(\alpha=1 / 2 n)($ cf. [13].)

\section{REFERENCES}

[1] J. Burbea, On metrics and distorsion theorem, Ann. of Math. Studies. No. 100 (1981), Princeton Univ. Press, 65-92.

[2] J. D'Atri, Holomorphic sectional curvature of bounded homogeneous domains and related questions, Trans. Amer. Math. Soc., 256 (1979), 405-412.

[3] K. Diederich and J. Fornaess, Comparison of the Bergman and Kobayashi metric, Math. Ann., 254 (1980), 257-260.

[4] L. Hörmander, An Introduction to Complex Analysis in Several Variables, Van Nostrand (1966).

[5] L. K. Hua, On the theory of automorphic functions of a matrix variable, Amer. J. Math., 66 (1944), 470-480.

[6] S. Kobayashi, Geometry of bounded domains, Trans. Amer. Math. Soc., 92 (1959), 267-290.

[7] _ Hyperbolic manifolds and holomorphic mappings, Pure and Appl. Math., 2, M. Dekker (1970).

[8] Intrinsic distances, measures and geometric function theory, Bull. Amer. Math. Soc., 82 (1976), 357-416.

[9] W. Rudin, Function Theory in the Unit Ball of $\mathbf{C}^{n}$, Grundlehren der Math. Wiss. 241, Springer Verlag, 1980.

[10] N. Sibony, A class of hyperbolic manifolds, Ann. of Math., Studies No. 100, Princeton Univ. Press. 
[11] M. Skwarczynski, Biholomorphic invariants related the Bergman functions, Dissertationes Math., 173 (1980).

[12] M. Suzuki, The holomorphic curvature of intrinsic metrics, Math. Rep. Toyama Univ., 4 (1981), 107-114.

[13] (1984), 249-256.

[14] The intrinsic metrics on the domains in $\mathbf{C}^{n}$, Math. Rep. Toyama Univ., 6 (1983).

Received July 18, 1983.

FACUlTy OF SCIENCE

TOYAMA UNIVERSITY

GOFUKU, TOYAMA

JAPAN 


\section{PACIFIC JOURNAL OF MATHEMATICS EDITORS}

DONALD BABBITT (Managing Editor)
University of California
Los Angeles, CA 90024
CHARLES R. DEPrIMA
California Institute of Technology
Pasadena, CA 91125
R. FINN
Stanford University
Stanford, CA 94305

\author{
HERMANN FLASCHKa \\ University of Arizona \\ Tucson, AZ 85721 \\ RAMESH A. GANGOLLI \\ University of Washington \\ Seattle, WA 98195 \\ ROBION KIRBY \\ University of California \\ Berkeley, CA 94720 \\ C. C. Moore \\ University of California \\ Berkeley, CA 94720
}

Hugo Rossi

University of Utah

Salt Lake City, UT 84112

H. SAMELSON
Stanford University
Stanford, CA 94305

HAROLD STARK

University of California, San Diego

La Jolla, CA 92093

\section{ASSOCIATE EDITORS}
R. ARens
E. F. BECKENBACH (1906-1982)
B. H. NeUmanN
F. WOLF
K. YOSHIDA

\begin{tabular}{ll}
\multicolumn{2}{c}{ SUPPORTING INSTITUTIONS } \\
UNIVERSITY OF ARIZONA & UNIVERSITY OF OREGON \\
UNIVERSITY OF BRITISH COLUMBIA & UNIVERSITY OF SOUTHERN CALIFORNIA \\
CALIFORNIA INSTITUTE OF TECHNOLOGY & STANFORD UNIVERSITY \\
UNIVERSITY OF CALIFORNIA & UNIVERSITY OF HAWAII \\
MONTANA STATE UNIVERSITY & UNIVERSITY OF TOKYO \\
UNIVERSITY OF NEVADA, RENO & UNIVERSITY OF UTAH \\
NEW MEXICO STATE UNIVERSITY & WASHINGTON STATE UNIVERSITY \\
OREGON STATE UNIVERSITY & UNIVERSITY OF WASHINGTON
\end{tabular}

The Supporting Institutions listed above contribute to the cost of publication of this Journal, but they are not owners or publishers and have no responsibility for its content or policies.

\footnotetext{
Mathematical papers intended for publication in the Pacıfic Journal of Mathematıcs should be in typed form or offset-reproduced (not dittoed), double spaced with large margins. Please do not use built up fractions in the text of the manuscript. However, you may use them in the displayed equations. Underline Greek letters in red, German in green, and script in blue. The first paragraph must be capable of being used separately as a synopsis of the entire paper. In particular it should contain no bibliographic references. Please propose a heading for the odd numbered pages of less than 35 characters. Manuscripts, in triplicate, may be sent to any one of the editors. Please classify according to the scheme of Math. Rev ews, Index to Vol. 39. Supply name and address of author to whom proofs should be sent. All other communications should be addressed to the managing editor, or Elaine Barth, University of California, Los Angeles, California 90024.

There are page-charges associated with articles appearing in the Pacific Journal of Mathematics. These charges are expected to be paid by the author's University, Government Agency or Company. If the author or authors do not have access to such Institutional support these charges are waived. Single authors will receive 50 free reprints; joint authors will receive a total of 100 free reprints. Additional copies may be obtained at cost in multiples of 50 .
}

The Pacific Journal of Mathematics is issued monthly as of January 1966. Regular subscription rate: $\$ 190.00$ a year (5 Vols., 10 issues). Special rate: $\$ 66.00$ a year to individual members of supporting institutions.

Subscriptions, orders for numbers issued in the last three calendar years, and changes of address should be sent to Pacific Journal of Mathematics, P.O. Box 969, Carmel Valley, CA 93924, U.S.A. Old back numbers obtainable from Kraus Periodicals Co., Route 100, Millwood, NY 10546.

The Pacific Journal of Mathematics at P.O. Box 969, Carmel Valley, CA 93924 (ISSN 0030-8730) publishes 5 volumes per year. Application to mail at Second-class postage rates is pending at Carmel Valley, California, and additional mailing offices. Postmaster: Send address changes to Pacific Journal of Mathematics, P.O. Box 969, Carmel Valley, CA 93924.

PUBLISHED BY PACIFIC JOURNAL OF MATHEMATICS, A NON-PROFIT CORPORATION Copyright $\odot 1985$ by Pacific Journal of Mathematics 


\section{Pacific Journal of Mathematics}

\section{Vol. 117, No. 2 \\ February, 1985}

Robert Walter Bagley, Ta-Sun Wu and J. S. Yang, On a class of topological groups more general than SIN groups ............... 209

Bruce Alan Barnes, Algebraic elements of a Banach algebra modulo an ideal ...............................................219

Howard D. Fegan and Peter Gilkey, Invariants of the heat equation .......233

Erica Flapan, Necessary and sufficient conditions for certain homology

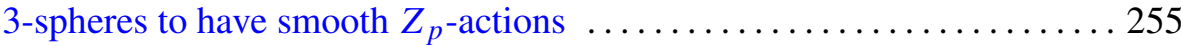

Philip R. Heath, Product formulae for Nielsen numbers of fibre maps . . . . 267

Derbiau Frank Hsu and A. Donald Keedwell, Generalized complete mappings, neofields, sequenceable groups and block designs. II ...... 291

Taqdir Husain, Orthogonal primitive idempotents and Banach algebras

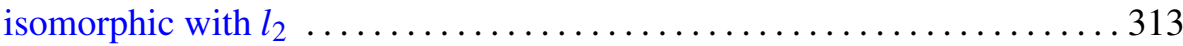

Gary M. Lieberman, Regularized distance and its applications . . . . . . . 329

William W. Menasco, Determining incompressibility of surfaces in alternating knot and link complements ............................ 353

Benjamin Muckenhoupt, Weighted reverse weak type inequalities for the Hardy-Littlewood maximal function $\ldots \ldots \ldots \ldots \ldots \ldots \ldots \ldots \ldots \ldots \ldots \ldots$

John Dacey O'Neill, Direct summands of direct products of slender

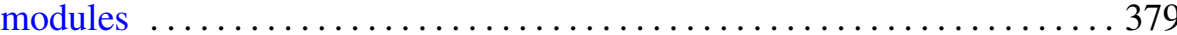

Andreas Rapp, Elimination of Malitz quantifiers in stable theories 387

Francisco José Ruiz, A unified approach to Carleson measures and $A_{p}$ weights

Hanamatagouda Pandappa Sankappanavar, Heyting algebras with dual

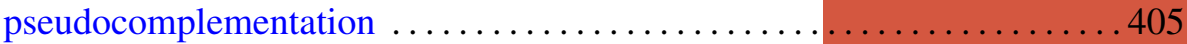

Annette Sinclair, Strong Carleman and strong uniform approximation . . . . 417

Masaaki Suzuki, The generalized Schwarz lemma for the Bergman metric

Brian Thorpe and Ludwig Tomm, Universal approximation by regular

weighted means 